\title{
Low-to-Moderate Alcohol Consumption May Be Safe When Taking Methotrexate for Rheumatoid Arthritis or Psoriasis
}

\author{
Bernard $\mathrm{Ng}^{1,2}$ \\ ${ }^{1}$ Houston Veterans Affairs HSR\&D Center of Excellence, Michael E. DeBakey Veterans Affairs Medical Center, Houston, USA; \\ ${ }^{2}$ Department of Medicine, Baylor College of Medicine, Houston, USA. \\ Email: Bernard.Ng@va.gov
}

Received May $4^{\text {th }}$, 2012; revised June $21^{\text {st }}, 2012$; accepted June $30^{\text {th }}, 2012$

\begin{abstract}
It is unclear if consumption of small to moderate amounts of alcohol is safe while taking methotrexate. We set out to determine whether there is an association between liver enzyme abnormalities and alcohol consumption in subjects taking methotrexate. The study sample was identified from the database of the South Central Veteran Affairs Healthcare Network, consisting of 10 hospitals in Texas, Oklahoma, Mississippi and Louisiana. From a cohort of 2443 eligible Veterans with rheumatoid arthritis and/or psoriasis taking methotrexate from 10/1/2007 to 9/30/2009, 120 cases with abnormal liver-enzyme elevation were randomly selected to compare with 120 controls. Data were collected from mailed survey forms that inquired about alcohol consumption habits, physicians' advice on alcohol, and methotrexate compliance. There was no significant difference in the number of non-drinkers and low-to-moderate alcohol drinkers between cases and controls $(\mathrm{p}=0.217)$. Few persons identified themselves as heavy drinkers. Our data suggest that it is likely safe for patients with rheumatoid arthritis and psoriasis to consume low-to-moderate amounts of alcohol while taking methotrexate. However, alternative methods to improve capturing alcohol consumption in heavy drinkers are needed for more comprehensive results.
\end{abstract}

Keywords: Methotrexate; Alcohol; Liver Enzymes; Rheumatoid Arthritis; Psoriasis

\section{Introduction}

In 2000, the National Household Survey on Drug Abuse estimated that 1.9 million Veterans reported heavy alcohol use [1]. The rate of past-month alcohol use was estimated to be similar in male Veterans and non-Veterans. In another report from the same source, men were twice as likely as women to be dependent on alcohol (10.5\% versus $5.1 \%$ ) [2]. With a $94 \%$ preponderance of men in the US Veteran population, the issue of alcohol use influencing compliance with a methotrexate (MTX) regimen and increasing risk of adverse effects is of greater importance for Veterans than it is for the general US population.

Rheumatoid arthritis (RA) and psoriasis are common conditions that afflict over 1.3 million and 4.5 million Americans, respectively [3,4]. The economic burden associated with such inflammatory conditions, with an average age of onset between 30 and 50, is exceptionally high because they affect working-age adults. Work disability accounts for much of the economic burden associated with RA and psoriatic arthritis. Therefore, early treatment of these chronic diseases is important to reduce the associated morbidity and mortality [4].

MTX has been used extensively in the treatment of RA and psoriasis since the early 1980s. It is a drug with a high benefit-to-risk ratio compared with other traditional disease-modifying, anti-rheumatic drugs (DMARDs). It has been estimated that about $20 \%-25 \%$ of psoriasis patients have been treated with MTX [5] and all new biological agents for RA rely on concomitant use of MTX for synergistic effects. Optimal dosing and patient compliance with the drug are important factors for successful treatment.

It is usually recommended that an individual abstain from or consume minimal amounts of alcohol while taking MTX because of potential liver toxicities [6]. This is a conservative recommendation that is based solely on expert opinion because there are no clinical data about the quantity of alcohol that can be safely consumed with MTX [6]. A review of 27 prospective studies estimated that, in subjects taking MTX, 20.2\% had at least one episode of elevated liver enzymes but that only $3.7 \%$ had to discontinue MTX because of liver toxicity [6]. Eleva- 
tion of liver enzymes is often transient and reverts to normal when alcohol or other hepatotoxic drugs (for example, non-steroidal anti-inflammatory drugs, statins, etc.) are stopped. Subsequently, MTX can be resumed at the same or a lower dose.

In the case of leflunomide, a DMARD that requires total abstinence from alcohol, one study reported that patients refused to take it when told that they needed to abstain from alcohol [8]. Unlike the treatment of HIV and diabetes, for which alcohol dependence has been shown to reduce medication compliance, there is little evidence that MTX compliance is affected by alcohol dependence $[9,10]$.

The purpose of this study was to examine whether there is an association between low-to-moderate alcohol consumption in patients taking MTX and elevated liver enzymes. The lack of an association would provide preliminary evidence that it might be safe for patients taking MTX to consume low-to-moderate amounts of alcohol.

\section{Methodology}

This study was approved by the Baylor College of Medicine Institutional Review Board and the Michael E. DeBakey Veterans Affairs Medical Center Research and Development Committee. In addition, because the study involved questions related to alcohol consumption, we also received a National Institutes of Health certificate of confidentiality.

\subsection{Study Setting}

The research was carried out with RA and psoriasis patients from the South Central Veteran Affairs Healthcare Network (SCVHCN), consisting of 10 VA hospitals in Texas, Oklahoma, Mississippi, and Louisiana.

Using an administrative database from the SCVHCN that contained patient demographics, diagnosis codes, drug prescriptions and laboratory results, we identified our incipient cohort as patients with RA and/or psoriasis who had been prescribed MTX for at least 6 months. Patients were eligible for inclusion if they met the following criteria: 1) had one ICD-9 code of 714.xx for RA or 696.xx for psoriasis between 10/1/2007 to 9/30/2009 and 2) had been taking MTX for at least 6 months. To ensure that we captured everyone who was prescribed at least 6 months of MTX, we searched drug prescriptions back to 4/1/2007. Exclusion criteria included having 1) abnormal liver enzymes prior to 10/1/2007 and 2) a known history of chronic viral hepatitis, autoimmune hepatitis or Sjögren's syndrome. A total of 2443 patients met these criteria.

\subsection{Study Population}

We used a case-control study design because of the small number of liver enzyme abnormalities in our cohort of RA and psoriasis patients on MTX. We defined cases as patients who had elevated liver enzymes (having at least one reading of high serum alanine aminotransferase (ALT) (i.e., greater than $80 \mathrm{U} / \mathrm{L}$ ) and/or high serum aspartate aminotransferase (AST) (i.e., greater than 120 U/L) while they were taking MTX within the study period. Controls had normal levels of ALT and AST while taking MTX.

From the incipient cohort of 2443 identified patients, we randomly selected 120 cases and 120 controls to whom a survey questionnaire, "Alcohol Use in Patients on Methotrexate" was mailed. This sample size was chosen based on the results of an earlier study for which the questionnaire was developed [11].

\subsection{Survey Questionnaire}

The "Alcohol Use in Patients on Methotrexate" survey for our study consisted of seven questions designed to estimate weekly alcohol consumption and to inquire about MTX compliance (see Appendix). The survey questions have been validated by a study in Norfolk, United Kingdom [11]. With permission from the author (Dr. Chris Deighton), we adapted the survey questions to be applicable to the US Veteran population.

Under the supervision of a psychometrician, we first conducted cognitive testing of the survey questions to ensure that the items were understandable and able to be answered by our Veteran population. In this part of the study, patients were not asked to answer the actual survey questions but asked to provide comments about the wording and meaning of the questions. In view of this brief survey, the psychometrician ascertained that four subjects would suffice for the cognitive testing process if there were a general consensus that the questions were clear and easy to understand. We enrolled four consecutive patients on MTX (with RA and/or psoriasis) from our rheumatology outpatient clinics.

For each of the seven questions in the survey, the subjects participating in the cognitive testing were asked to comment on the followings:

1) How important do you feel this question is?

2) Describe what comes to mind when you hear this question.

3) Do you get a clear picture in your mind right away of what your answer would be?

a) If so, what makes this question clear?

b) If not, what makes this question unclear?

4) How would you ask this question to make it clearer?

5) How hard would it be to answer this question?

a) If you think it would be hard, what makes it hard?

b) What would make it easier to answer?

6) How comfortable would you feel answering this 
question?

a) If comfortable, what makes you feel comfortable about answering this question?

b) If uncomfortable, what makes you feel uncomfortable about answering this question?

\subsection{Survey Administration}

The survey and a consent form were mailed to study subjects. Subjects were instructed in the consent form to sign the consent form, complete the survey, and return the documents to us in a self-addressed, stamped envelope. One week after mailing out the surveys, we contacted subjects by telephone to ask if they had questions about the survey. Phone interviews to obtain survey answers or verbal consents were not allowed by our IRB.

\subsection{Data Analysis}

Using the Chi-square test, we examined whether the percentage of patients with abnormal liver enzymes in our eligible study population differed among patients with RA only, psoriasis only, and those with both RA and psoriasis. We also conducted a multivariable logistic regression analysis in which the dependent variable was whether or not the patient had abnormal liver enzymes. Gender and disease diagnoses were used as independent variables.

We next compared the cases and controls to whom surveys were sent on their mortality rates, survey response rates, demographic characteristics and medication use. For continuous variables such as age, we used the nonparametric Wilcoxon two-sample test to compare cases and controls. For categorical variables, we used the Chi-square test. For survey questions with low response rates, we did not conduct formal statistical tests but instead reported only the numbers and percentages of patients responding to the survey items. When examining questions related to MTX compliance and doctor's advice, we categorized patients into three categories: nondrinkers, low-to-moderate drinkers, and heavy drinkers. From the many possible definitions of moderate alcohol consumption, we selected the widely accepted-National Institute on Alcohol Abuse and Alcoholism (NIAAA) definition of 7 - 14 standard drinks per week for men and up to 1 - 7 standard drinks per week for women. We use the definition of standard drink as any drink that contains about $14 \mathrm{~g}$ pure alcohol (12 oz. of beer/8 - 9 oz. of malt liquor $/ 5 \mathrm{oz}$. of table wine/1.5 oz. of hard liquor or spirits) [12,13]. We tabulated the responses of the cases and controls as to whether they were drinkers, what their level of alcohol consumption was, and what their views of alcohol consumption and use of MTX were.

\section{Results}

\subsection{Characteristics of Study Population Taken from the SCVHCN Administrative Database}

Within our regional VA administrative database, there were $2443 \mathrm{RA}$ and psoriasis patients meeting our study criteria. Among these, there were 179 subjects (7.3\%) with at least one abnormal liver function test during the study period. Table 1 shows significant differences among patients with RA only, those with psoriasis only, and those with both conditions as to whether they had abnormal liver enzymes $(p<0.001)$. In terms of patients with RA only, 6.3\% had abnormal levels, compared with $10.8 \%$ of those with psoriasis only and $13.6 \%$ of those with both diseases.

In the multivariable logistic regression model, we found that psoriasis only patients and patients with both diseases were more likely than RA only patients to have abnormal liver enzymes (odds ratio for psoriasis only patients $=1.76,95 \%$ CI: $1.21-2.55$ and odds ratio for patients with both diseases $=2.31$, 95\% CI: 1.28 - 4.18). Males were more likely to have abnormal liver enzymes than females, but this did not reach statistical significance (odds ratio $=2.21,95 \%$ CI: $0.89-5.48$ ).

\subsection{Cognitive Testing Phase of the Survey}

Four consecutive patients (two men and two women) participated in the cognitive testing of the survey questions. All four patients felt that all seven questions were important and clearly worded. They also reported that they felt comfortable answering these questions.

Table 1. Summary of our study population taken from the SCVHCN administrative database.

\begin{tabular}{|c|c|c|}
\hline Disease & $\begin{array}{l}\text { Abnormal liver } \\
\text { enzymes (\%) }\end{array}$ & $\begin{array}{l}\text { Normal liver } \\
\text { enzymes (\%) }\end{array}$ \\
\hline RA only $(n=1959)$ & 6.3 & 93.7 \\
\hline Males $(\mathrm{n}=1833)$ & 6.6 & 93.4 \\
\hline Females $(n=126)$ & 3.2 & 96.8 \\
\hline Psoriasis only $(\mathrm{n}=381)$ & 10.8 & 89.2 \\
\hline Males $(\mathrm{n}=366)$ & 10.9 & 89.1 \\
\hline Females $(n=15)$ & 6.7 & 93.3 \\
\hline Both diseases $(n=103)$ & 13.6 & 86.4 \\
\hline Males $(n=98)$ & 14.3 & 85.7 \\
\hline Females $(\mathrm{n}=5)$ & 0.0 & 100.0 \\
\hline
\end{tabular}

Overall: $x_{2}^{2}=15.4(\mathrm{p}<0.001)$; Males: $x_{2}^{2}=15.0(\mathrm{p}=0.001)$; Females: Fisher's exact test $(\mathrm{p}=0.53)$. 


\subsection{Survey Results}

At the time of the survey, 14 patients in the case group and four patients in the control group were unreachable. Table 2 compares the patients' demographics. There were no significant differences in the distribution of age, gender and diagnoses between cases and controls. The use of related drugs such as folic acid and other oral DMARDs was similar in both groups. The average daily prednisone dose was significantly higher in the cases. Although the percentage of patients ever using $10 \mathrm{mg}$ or more of prednisone daily was higher amongst the cases, this did not reach statistical significance. We would expect MTX to be a confounding factor for abnormal raised liver enzymes if average MTX dose is higher amongst the cases. However, average MTX dose was significantly higher in the control group. This could be due to MTX dose being reduced when abnormal levels of liver enzymes were found.

With regard to the differences in patients' compliance with MTX and physicians' advice on alcohol consumption, $70.3 \%$ of non-drinkers reported to have never missed a dose of MTX compared to $24.3 \%$ of low-tomoderate drinkers and $5.4 \%$ of heavy drinkers. Most of the non-drinkers remembered being advised by their physicians to avoid alcohol completely (60\%) while only $10 \%$ of heavy drinkers remembered hearing this advice. Among those who were told that they could consume small amounts of alcohol (up to 2 drinks per day for men and 1 drink per day for women) while on MTX, 67\% were low-to-moderate drinkers and 33\% were heavy drinkers. None of the non-drinkers remembered hearing the advice that low-to-moderate alcohol consumption was acceptable.

Table 2. Comparison of cases and controls to whom surveys were sent on their response rate, demographic characteristics and use of related medicines.

\begin{tabular}{|c|c|c|c|}
\hline & Cases $(n=106)$ & Controls $(n=116)$ & $\mathrm{p}$-value \\
\hline Response rate (\%) & 24.5 & 23.3 & $0.88^{\sharp}$ \\
\hline Average age (as of 6/1/2010) & 64.0 & 65.1 & $0.37^{*}$ \\
\hline Sex (\% male) & 91.5 & 92.2 & $0.85 \S$ \\
\hline RA only (\%) & 68.9 & 76.7 & \\
\hline Psoriasis only (\%) & 24.5 & 14.7 & $0.17 \S$ \\
\hline RA and psoriasis (\%) & 6.6 & 8.6 & \\
\hline Average methotrexate dose (per week) & 13.4 & 14.7 & $0.03^{*}$ \\
\hline$\%$ on folic acid & 91.5 & 91.4 & $0.97 \S$ \\
\hline $\begin{array}{l}\text { Concomitant use of other DMARDS (\% of patients on the } \\
\text { following drugs): }\end{array}$ & 14.2 & 12.9 & $0.79 \S$ \\
\hline a) Leflunomide & 16.0 & 11.2 & $0.29 \S$ \\
\hline b) Sulfasalazine & 31.1 & 37.9 & $0.29 \S$ \\
\hline c) Hydroxychloroquine & & & \\
\hline $\begin{array}{l}\% \text { who had been on } 10 \mathrm{mg} \text { or more of prednisone during } \\
\text { study }\end{array}$ & 46.3 & 34.5 & $0.07 \S$ \\
\hline Average prednisone dose daily (mg) & 15.4 & 11.8 & $<0.01^{*}$ \\
\hline$\%$ with concomitant use of statins & 60.4 & 56.0 & $0.51^{\S}$ \\
\hline$\%$ with concomitant use of retinols & 3.8 & 5.2 & $0.75^{\sharp}$ \\
\hline
\end{tabular}

ॠ Fischer’s exact test; ${ }^{*}$ Wilcoxon two sample test; ${ }^{\S}$ Chi-square. 
The amount of alcohol consumed between the cases and controls were not significantly different $(p=0.17)$. There was also no significant difference when we compared the distribution among cases and controls of nondrinkers with light-to-moderate drinkers and heavy drinkers ( $p=0.37$, Fisher's exact).

All heavy alcohol drinkers reported that knowing lowto-moderate alcohol consumption is acceptable when taking MTX would not change their methotrexate compliance. The heavy alcohol drinkers also reported no reduction in the amount of alcohol consumed since they started MTX. However, some of those who consume low-to-moderate amounts of alcohol did report that they might improve MTX compliance if they knew that lowto-moderate alcohol consumption is acceptable when taking MTX.

\section{Discussion}

There is a lack of information about the amount of alcohol that can be safely consumed by patients with RA or psoriasis while taking MTX. Due to concerns for increased risk of hepatotoxicity, many physicians prefer that patients adhere to strict alcohol abstinence. However, despite the high rates of alcohol use in a predominantly male VA population, elevated liver enzymes among patients with RA or psoriasis while on MTX are lower (7.3\%) compared with studies done on the general population $(9.3 \%$ and $17.0 \%)$ [11,14]. Our study used a casecontrol design because of the low rate of elevated liver enzymes while on MTX among our study population of patients with RA or psoriasis.

Elevations of liver enzymes in patients taking MTX are typically more frequently observed in patients with psoriasis than in patients with RA. This is consistent with previous findings that MTX-associated hepatotoxicity is more often observed in patients with psoriasis than in patients with RA [15]. Such observations had been attributed to the greater preponderance of men with psoriasis compared with RA and higher alcohol consumption by men. However, in our study population which has a low number of females, there is a no difference in the proportion of males and females between the two diagnoses. Yet we still have significantly higher abnormal liver enzyme occurrences among psoriatic patients. In addition, when we looked only at males, the occurrence of abnormal liver enzymes was still significantly higher in psoriasis only patients compared with RA only patients. This point is supported by our logistic regression model where we found increased odds of having abnormal liver enzymes in patients with psoriasis only and with both diseases compared with RA only. The increased odds remained significant even when corrected for gender. Therefore, there could be other possible rea- sons (e.g., genetic factors) because gender differences between the two diagnoses cannot fully explain these observations.

Studies using a large database have the advantages of being cost effective, convenient and able to provide a larger study population which would be difficult to achieve with other study methods [16-22]. The VA database is the largest healthcare administrative database in the United States and is a useful resource for epidemiologic studies [23]. However, epidemiologic studies using databases rely heavily on the accuracy of diagnostic coding, and their reliability depends on the physicians' ability to make an accurate diagnosis. Singh et al. have pointed out that using only diagnosis code 714.xx to define a prevalent sample of RA patients has a low specificity of $55 \%$,with an unacceptably high false-positive rate of $34 \%$ [24]. However, we have further added the criterion of being on MTX for at least 2 years. This criterion, according to Singh et al., would increase the specificity to an acceptable level of $83 \%-97 \%$ and increase the positive predictive value from $81 \%-97 \%$. In a report of another study, Singh et al. indicated that using the ICD-9 diagnosis code to screen for psoriatic arthritis is both sensitive (57\% - 100\%) and specific (98\% - 100\%) [25]. However, adding the DMARD criterion to define the cohort could result in lowering the sensitivity. Our study design omitted patients who had discontinued MTX within 6 months for various adverse effects, such as abnormal liver function tests or drug hypersensitivity. We excluded cases with less than 6 months of MTX use because of the following:

1) It could indicate that MTX was discontinued upon the physician's realization of a misdiagnosis of RA or psoriasis;

2) Elevated liver enzymes for other reasons could have preceded initiation of MTX, and the prescribing physician would have discontinued MTX when he/she realized this.

A limitation resulting from the exclusion of such cases is that true cases may have been omitted, such as those of patients who consumed alcohol and developed elevated liver enzymes because they were unable to reduce alcohol intake, and so the prescribing physician discontinued MTX within the first 6 months. Another limitation includes our inability to capture drug prescription information when patients obtained drugs from outside the VA system-for example, aspirin and other non-steroidal anti-inflammatory drugs which may be cheaper to purchase from a retail pharmacy. In addition, we have a predominantly male Veteran population which reduces the ability to extrapolate our findings to the general population.

Our data suggest that liver enzyme elevations in subjects with RA or psoriasis taking MTX are unlikely to be affected 
by low-to-moderate amounts of alcohol consumption.

\section{Acknowledgments}

This work was supported by a local grant from the Houston Center for Quality of Care and Utilization Studies (CF.HQU.LFP.0110.000-00.B_N) and also partly supported by the Houston Veterans Affairs Health Services Research and Development Center of Excellence (HFP 90-020). The views expressed are those of the authors and do not necessarily represent those of the Department of Veterans Affairs/Baylor College of Medicine.

The authors would like to thank Sonora Hudson for editing the manuscript, Crystal Booker for helping to contact the survey participants and tabulation of results, Dr. Fawad Aslam for helping with initial study design and pre-testing phase of the survey, and the Veterans Affairs South Central Mental Illness Research, Education and Clinical Center for providing help from Dr. Adam Kelly with the psychometrics of the survey form and from Annette Walder with data organization.

\section{REFERENCES}

[1] Office of Applied Studies, "Alcohol Use among Veterans,” Office of Applied Studies, Rockville, 2001.

[2] Office of Applied Studies, "Gender Differences in Alcohol Use and Alcohol Dependence or Abuse: 2004 and 2005,” Office of Applied Studies, Rockville, 2007.

[3] R. S. Stern, T. Nijsten, S. R. Feldman, et al., "Psoriasis Is Common, Carries a Substantial Burden Even When Not Extensive, and Is Associated with Widespread Treatment Dissatisfaction," Journal of Investigative Dermatology Symposium, Vol. 9, No. 2, 2004, pp. 136-139. doi:10.1046/j.1087-0024.2003.09102.x

[4] C. G. Helmick, D. T. Felson, R. C. Lawrence, et al., "Estimates of the Prevalence of Arthritis and Other Rheumatic Conditions in the United States. Part I," Arthritis \& Rheumatism, Vol. 58, No. 1, 2008, pp. 15-25. doi:10.1002/art.23177

[5] T. Nijsten, D. J. Margolis, S. R. Feldman, et al., "Traditional Systemic Treatments Have Not Fully Met the Needs of Psoriasis Patients: Results from a National Survey," Journal of the American Academy of Dermatology, Vol. 52, No. 3, 2005, pp. 434-444.

[6] J. M. Kremer, G. S. Alarcon, R. W. Lightfoot Jr., et al., "Methotrexate for Rheumatoid Arthritis. Suggested Guidelines for Monitoring Liver Toxicity. American College of Rheumatology," Arthritis \& Rheumatism, Vol. 37, No. 3, 1994, pp. 316-328. doi:10.1002/art.1780370304

[7] C. Salliot and D. van der Heijde, "Long Term Safety of Methotrexate Monotherapy in Rheumatoid Arthritis Patients: A Systematic Literature Research,” Annals of the Rheumatic Diseases, Vol. 68, No. 7, 2008, pp. 11001104.

[8] S. Rajakulendran and C. Deighton, “Do Guidelines for the Prescribing and Monitoring of Leflunomide Need to Be Modified?” Rheumatology, Vol. 43, No. 11, 2004, pp. 1447-1448. doi:10.1093/rheumatology/keh348

[9] C. L. Bryson, D. H. Au, H. Sun, et al., "Alcohol Screening Scores and Medication Nonadherence,” Annals of Internal Medicine, Vol. 149, No. 11, 2008, pp. 795-804.

[10] J. Dunbar-Jacob, J. L. Holmes, S. Sereika, et al., "Factors Associated with Attrition of African Americans during the Recruitment Phase of a Clinical Trial Examining Adherence among Individuals with Rheumatoid Arthritis," Arthritis \& Rheumatism, Vol. 51, No. 3, 2004, pp. 422428. doi:10.1002/art.20411

[11] S. Rajakulendran, K. Gadsby and C. Deighton, "Rheumatoid Arthritis, Alcohol, Leflunomide and Methotrexate. Can Changes to the BSR Guidelines for Leflunomide and Methotrexate on Alcohol Consumption Be Justified?” Musculoskeletal Care, Vol. 6, No. 4, 2008, pp. 233-245. doi:10.1002/msc. 135

[12] US Department of Health and Human Services, "Helping Patients Who Drink Too Much: A Clinician's Guide,” NIAAA Publications Distribution Center, Rockville, 2005, p. 24.

[13] M. C. Dufour, "What Is Moderate Drinking? Defining 'Drinks' and Drinking Levels,” Alcohol Research \& Health, Vol. 23, No. 1, 1999, pp. 5-14.

[14] K. Visser and D. M. Van der Heidje, "Incidence of Liver Enzyme Elevations and Liver Biopsy Abnormalities during Methotrexate Treatment in Rheumatoid Arthritis: A Systematic Review of the Literature," Arthritis \& Rheumatism, Vol. 58, 2008, p. S557.

[15] R. E. Kalb, B. Strober, G. Weinstein, et al., "Methotrexate and Psoriasis: 2009 National Psoriasis Foundation Consensus Conference," Journal of the American Academy of Dermatology, Vol. 60, No. 5, 2009, pp. 824-837. doi:10.1016/j.jaad.2008.11.906

[16] R. A. Bright, J. Avorn and D. E. Everitt, "Medicaid Data as a Resource for Epidemiologic Studies: Strengths and Limitations,” Journal of Clinical Epidemiology, Vol. 42, No. 10, 1989, pp. 937-945. doi:10.1016/0895-4356(89)90158-3

[17] J. P. Burke, H. H. Tilson and R. Platt, "Expanding Roles of Hospital Epidemiology: Pharmacoepidemiology,” Infection Control and Hospital Epidemiology, Vol. 10, No. 6, 1989, pp. 253-254. doi:10.1086/646016

[18] I. K. Crombie, "The Role of Record Linkage in PostMarketing Drug Surveillance,” British Journal of Clinical Pharmacology, Vol. 22, No. 1, 1986, pp. 77S-82S. doi:10.1111/j.1365-2125.1986.tb02987.x

[19] G. A. Faich, "Record Linkage for Postmarketing Surveillance," Clinical Pharmacology and Therapeutics, Vol. 46, No. 4, 1989, pp. 479-480. doi:10.1038/clpt.1989.169

[20] W. A. Ray and M. R. Griffin, "Use of Medicaid Data for Pharmacoepidemiology," American Journal of Epidemiology, Vol. 129, No. 4, 1989, pp. 837-849.

[21] S. Shapiro, "The Role of Automated Record Linkage in the Postmarketing Surveillance of Drug Safety: A Critique,” Clinical Pharmacology and Therapeutics, Vol. 46, 
No. 4, 1989, pp. 371-386. doi:10.1038/clpt.1989.154

[22] H. H. Tilson, "Pharmacoepidemiology: The Lessons Learned; the Challenges Ahead," Clinical Pharmacology and Therapeutics, Vol. 46, No. 4, 1989, p. 480. doi:10.1038/clpt.1989.170

[23] E. J. Boyko, T. D. Koepsell, J. M. Gaziano, et al., "US Department of Veterans Affairs Medical Care System as a Resource to Epidemiologists," American Journal of Epidemiology, Vol. 151, No. 3, 2000, pp. 307-314. doi:10.1093/oxfordjournals.aje.a010207
[24] J. A. Singh, A. R. Holmgren and S. Noorbaloochi, “Accuracy of Veterans Administration Databases for a Diagnosis of Rheumatoid Arthritis," Arthritis Care \& Research, Vol. 51, No. 6, 2004, pp. 952-957. doi:10.1002/art.20827

[25] J. A. Singh, A. R. Holmgren, H. Krug, et al., “Accuracy of the Diagnoses of Spondylarthritides in Veterans Affairs Medical Center Databases," Arthritis Care \& Research, Vol. 57, No. 4, 2007, pp. 648-655. doi:10.1002/art.22682

i) Number of $12 \mathrm{oz}$. drinks consumed in one week:

\section{APPENDIX: Questions in Alcohol Use in Patients on Methotrexate Survey}

Adapted from a study in Norfolk, United Kingdom with permission from the author (Dr. Chris Deighton) [11].

1) How often do you miss your Methotrexate dose?

a) In ever miss a dose.

b) On average, I miss a dose once in six months.

c) On average, I miss a dose once in four months.

d) On average, I miss a dose once in two months.

e) On average, I miss a dose every month.

f) I am not sure about this.

2) What did your doctor tell you about drinking alcohol when you were started on Methotrexate?

a) Avoid alcohol completely.

b) Drink as you like.

c) No advice was given.

d) Cannot remember.

e) Not applicable to me since I do not drink alcohol.

3) Were you told that drinking alcohol up to 2 standard drinks per day for men and 1 standard drink per day for women is ok?

a) Yes.

b) No.

c) Cannot remember.

4) Do you drink any alcohol? (Please include social drinking as well)

a) Yes.

b) No.

If you answer no, then you need not answer any further questions.

5) Please average your alcohol intake over the last 6 months. Please include episodes, if any, of binge drinking as well. You may choose as many answers as they apply to you.

a) If you drink beer, please state the average number of drinks: ii) Number of 16 oz. drinks consumed in one week:

iii) Number of $22 \mathrm{oz}$. drinks consumed in one week:

iv) Number of 40 oz. drinks consumed in one week:

v) Other: Private Stock, Camo 40, King Cobra and others) please state the average number:

i) Number of 12 oz. drinks consumed in one week:

ii) Number of 16 oz. drinks consumed in one week:

iii) Number of 20 oz. drinks consumed in one week:

iv) Number of $40 \mathrm{oz}$. drinks consumed in one week:

v) Other:

c) If you drink hard liquor/spirits (80-proof drinks) (example: brandy, gin, vodka, whiskey) please state the average number:

i) Number of shots $(1.5 \mathrm{oz}$.) consumed in one week:

ii) Number of Pint (16 oz.) drinks consumed in one week:

iii) Number of Fifth (25 oz.) drinks consumed in one week:

iv) Number of 1.75 liters (59 oz.) drinks consumed in one week:

v) Number of Mixed drink (please specify):

If you drink table wine, fortified wine, Cordial, Liqueurs (example Sherry, Port) please state the average number:

i) Number of servings/single bottle/glasses (5 oz.) b) If you drink malt liquor (example: Forty, Colt 45 , 
glasses consumed in one week:

ii) Number of standard $750 \mathrm{ml}$ (25oz.) bottle consumed in one week:

iii) Other:

6) The current recommendation is to avoid alcohol while taking Methotrexate. If the recommendation were changed such that alcohol drinking was allowed while taking Methotrexate, how would that affect your Methotrexate intake? a) I would use my medicine more regularly.

b) I would use my medicine less regularly.

c) It would not make a difference.

7) How has your alcohol drinking changed since you have been on Methotrexate?

a) I have been drinking more alcohol.

b) I have been drinking less alcohol.

c) I have completely stopped drinking alcohol.

d) There has been no change in my drinking habits. 